\title{
Ileocecal ulceration and granulomatous ileitis as an unusual presentation of typhoid fever
}

\author{
Christopher Cheung BSc, Hayley Merkeley BSc MD, Jocelyn A. Srigley MD, Baljinder Salh MD, \\ Douglas Webber MD, Stephane Voyer MD
}

Competing interests: None declared.

This article has been peer reviewed.

\section{Correspondence to:} Christopher Cheung, cccheung@interchange.ubc. ca

CMAJ 2012. DOI:10.1503 /cmaj.120714

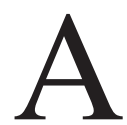
55-year-old man presented to hospital with a 2-week history of cyclical fevers, chills, night sweats, decreased appetite and weight loss. Originally from India, the patient had lived in Canada for 25 years, but he had recently returned from a 2-month vacation in Punjab, India. He had not received any vaccinations before travel, nor had he taken any malaria prophylaxis. The patient reported having consumed locally prepared foods and water from untreated sources.

The patient started having fevers $\left(40^{\circ} \mathrm{C}\right)$, rigors, night sweats and nonbloody diarrhea during the last week of his vacation. Upon returning to Canada, his diarrhea resolved, and he was given a 3-day course of oral ciprofloxacin for persistent symptoms (i.e., cyclical fevers and night sweats). Despite taking his antibiotic medication, the patient's symptoms continued; he presented to hospital 1 week after returning to Canada.

The patient's medical history included type 2 diabetes that was being treated with oral hypoglycemic agents. There was no personal or family history of tuberculosis, sarcoidosis, inflammatory bowel disease, colorectal cancer or HIV. There were no other symptoms of infection, and the patient had not recently been sexually active.

A physical examination showed tachycardia and a fever of $39.6^{\circ} \mathrm{C}$. We found no jaundice or palpable lymphadenopathy. An abdominal examination showed mild periumbilical tenderness, but no appreciable mass or organomegaly.

The results of laboratory investigations showed that the patient had a normal white blood cell count $\left(7.8 \times 10^{6}\right.$ cells $\left./ \mathrm{L}\right)$, but that his liver enzyme (ala-

\section{KEY POINTS}

- Typhoid fever is an important consideration in patients with fever of unknown origin, particularly in travellers returning from endemic areas.

- It commonly affects the lymphoid tissue of the ileum, appendix and right colon, with ulceration and bleeding seen on colonoscopy.

- It may be associated with granulomatous ileitis, although intestinal tuberculosis needs to be ruled out. nine transaminase 126 [normal 10-65) U/L, aspartate transaminase 81 [normal 10-38] U/L), $l$-lactate dehydrogenase (665 [normal 90-240] U/L) and Creactive protein $(85$ [normal $<3.1] \mathrm{mg} / \mathrm{L})$ levels were elevated. Cultures of the patient's stool and blood showed no growth. Smears for ova, parasites and malaria were negative, as were the results of serologic tests for dengue, HIV, syphilis, and hepatitis B and $\mathrm{C}$.

Computed tomography of the patient's abdomen showed borderline splenomegaly with periaortic, retroperitoneal and mesenteric lymphadenopathy. We saw mucosal thickening at the ileocecal junction. We gave the patient piperacillintazobactam (3.375 g intravenously, every $6 \mathrm{~h}$ ) for presumed intra-abdominal infection.

Subsequent colonoscopy showed ulcerations throughout the colon (Figure 1), raising the possibility of tuberculosis, Crohn disease or Yersinia infection. We thus changed the patient's medication to doxycycline (100 mg orally, twice daily) and gentamicin (350 mg intravenously, once daily).

Biopsies of the patient's ileum and cecum showed histiocyte-rich granulomatous changes (Figure 2) with areas of central necrosis. The possibility of mycobacterial infection was ruled out with further investigations: a tuberculin skin test, two Ziehl-Neelsen stains of the ileal and cecal tissue samples and a polymerase chain reaction (PCR) test for Mycobacterium species returned negative results. Cultures of the cecal aspirate showed no growth of Mycobacterium tuberculosis. In addition, cold enrichment culture for Yersinia on stool specimens showed no growth.

One week after the patient's admission to hospital, a repeat stool culture grew Salmonella enterica serotype Typhi (susceptible to ampicillin, ceftriaxone and trimethoprim/ sulfamethoxazole), confirming a diagnosis of typhoid fever. We gave the patient ceftriaxone, and his symptoms improved quickly; after $48 \mathrm{~h}$, the patient had been afebrile for $24 \mathrm{~h}$. 


\section{Discussion}

Typhoid fever is a systemic illness predominantly characterized by fever and abdominal pain. ${ }^{1}$ Humans are the sole reservoir of the responsible organism, S. enterica serotype Typhi. In Ontario alone, the incidence is about 50-73 cases per year. Most cases occur in travellers returning from endemic areas, such as India, Mexico and the Philippines., Vaccination with the Vi or attenuated Ty21a vaccine before travel to endemic areas may protect against typhoid fever; however, travellers should be advised to maintain food and water precautions. ${ }^{2}$

Symptoms of typhoid fever typically develop 5-21 days after ingesting contaminated food or water. Classical descriptions of acute typhoid fever identify a stepwise progression of clinical findings over 3 weeks, beginning with fever and relative bradycardia within the first week of illness. ${ }^{4}$ Abdominal pain and a rash characterized by rose or salmon-coloured macules on the trunk and abdomen may develop during the second week. Hepatosplenomegaly, intestinal bleeding and perforation secondary to ileocecal lymphatic hyperplasia may occur during the third week. ${ }^{4}$
Presentation varies with age, geography, serotype and the size of inoculum. ${ }^{5}$ It is diagnosed when $S$. enterica serotype Typhi is cultured from blood, stool, urine, rose spots, duodenal contents or bone marrow from patients presenting with a concomitant clinical illness consistent with typhoid fever. $^{4}$

Typhoid fever commonly affects the lymphoid tissue within the ileum, appendix and right colon. Histologically, the affected areas are characterized by aphthous ulcers, hyperplasia of Peyer's patches, crypt abscesses and marked architectural distortion that may lead to fullthickness ulceration and necrosis. ${ }^{4}$ Common colonoscopic manifestations of typhoid fever include punched-out ulcerations of various sizes, edematous hyperemic mucosal patches and active bleeding. ${ }^{6}$ We saw similar ileocecal and colonic ulcerations on colonoscopy; however, we also found granulomas on biopsy in the absence of mycobacterial infection.

\section{Intestinal granulomas}

Typical diseases that produce intestinal granulomas include abdominal tuberculosis, sarcoidosis, Crohn disease and, occasionally, Yersinia infec-

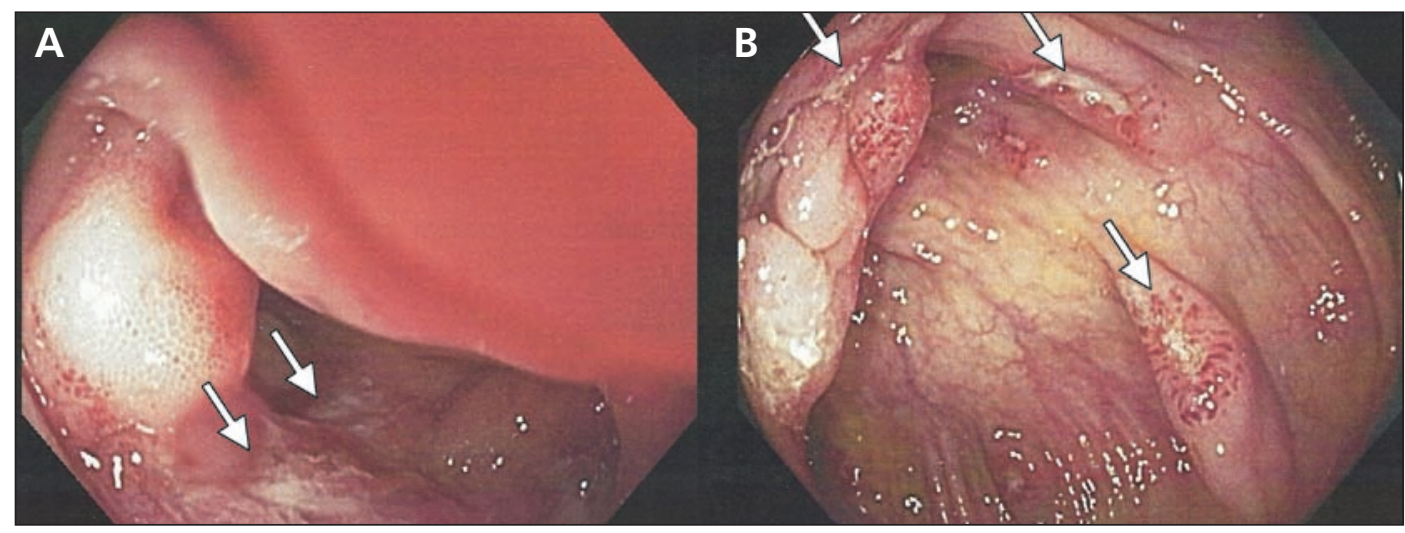

Figure 1: Colonoscopic images of the terminal ileum (A) and proximal ascending colon (B) showing discrete transverse ulcers with mildly hypertrophic erythematous margins (arrows).

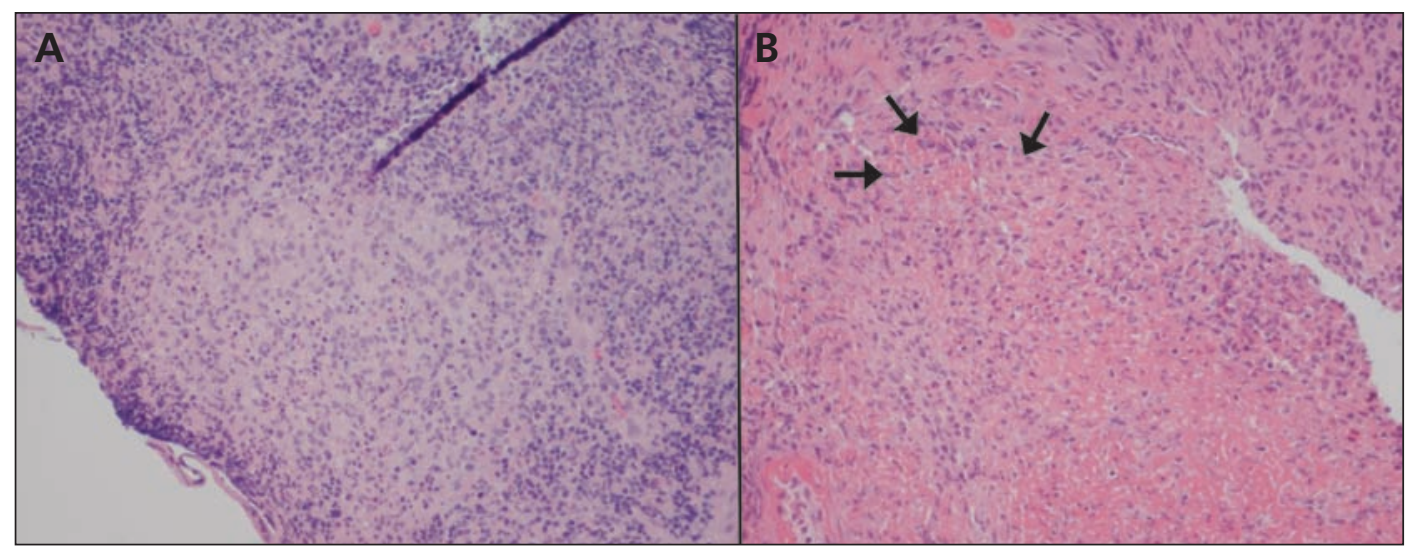

Figure 2: Microscopy (hematoxylin and eosin stain, 200x magnification) of ileal mucosal biopsy (A) and cecal mucosal biopsy (B) showing histiocyte-rich granulomatous changes with central necrosis (arrows). 
tion. $^{7}$ Although intestinal granulomas are rarely seen in typhoid fever, extraintestinal granulomatous changes have been documented (Appendix 1, available at www.cmaj.ca/lookup/suppl/doi :10.1503/cmaj.120714/-/DC1). Reports of granulomas in the liver, bone marrow and spleen with typhoid fever have been published previously. ${ }^{8}$ An account of cutaneous granulomata associated with the typhoid-paratyphoid heat-killed vaccine has also been reported. ${ }^{9}$

Previously reported histopathologic findings associated with typhoid enteritis include hypertrophy of the ileal mucosa and neutrophil-poor, monocyte/macrophage-rich mucosal infiltrates. ${ }^{10}$ However, there are only 2 case reports of intestinal granulomatous changes in patients with typhoid fever. Reyes and colleagues described colonic granulomas with large multinucleated cells in a patient whose bone marrow tested positive for $S$. enterica serotype Typhi. ${ }^{11}$ Similarly, Bharadwaj and coauthors described necrotizing and non-necrotizing granulomas in a biopsy from the ileocolic junction of a patient confirmed to have $S$. enterica serotype Typhi. ${ }^{12}$

Neither of these previous case reports explicitly excluded the possibility of intestinal tuberculosis as a cause of the granulomatous changes seen, either with PCR testing or by culturing mycobacteria. PCR testing for mycobacteria is more sensitive than traditional microscopy, with a sensitivity for detecting intestinal tuberculosis of $86 \%$, and a specificity approaching $100 \% .^{13}$ In our patient's case, we ruled out active mycobacterial infection after samples of ileal and cecal tissue showed negative results for stains for acid-fast bacilli and PCR testing for mycobacteria. Furthermore, our patient had a negative result on his tuberculin skin test. Discrete intestinal granulomatous changes in the context of typhoid fever and the absence of mycobacterial disease is a novel finding.

\section{Conclusion}

This case report shows the importance of considering typhoid fever as a cause of ileocecal ulceration and granulomatous changes in the gastrointestinal tract of patients presenting with a fever of unknown origin, particularly if they are returning from areas where $S$. enterica serotype Typhi is prevalent.

\section{References}

1. Parry CM, Hien TT, Dougan G, et al. Typhoid fever. $N$ Engl J Med 2002;347:1770-82

2. Immunization NACo. Part 4: Active immunizing agents: typhoid vaccine. In: Canadian immunization guide: 2006 . 7th ed. Ottawa (ON): Public Health Agency of Canada; 2006.

3. Morris SK, Richardson SE, Sauve LJ, et al. Increasing fluoroquinolone resistance in Salmonella typhi in Ontario, 2002-2007. Am J Trop Med Hyg 2009;80:1012-3.

4. Lamps LW. Infective disorders of the gastrointestinal tract. Histopathology 2007;50:55-63.

5. Huang DB, DuPont HL. Problem pathogens: extra-intestinal complications of Salmonella enterica serotype Typhi infection. Lancet Infect Dis 2005;5:341-8.

6. Lee JH, Kim JJ, Jung JH, et al. Colonoscopic manifestations of typhoid fever with lower gastrointestinal bleeding. Dig Liver Dis 2004;36:141-6.

7. AbdullGaffar B. Granulomatous diseases and granulomas of the appendix. Int J Surg Pathol 2010;18:14-20.

8. Mert A, Tabak F, Ozaras R, et al. Typhoid fever as a rare cause of hepatic, splenic, and bone marrow granulomas. Intern Med 2004;43:436-9.

9. Schneidman HM, Steinberg J. Reactions to intradermal vaccinations. A report of three cases of foreign body granulomas. Calif Med 1964;100:287-9.

10. Kraus MD, Amatya B, Kimula Y. Histopathology of typhoid enteritis: morphologic and immunophenotypic findings. Mod Pathol 1999;12:949-55.

11. Reyes E, Hernandez J, Gonzalez A. Typhoid colitis with massive lower gastrointestinal bleeding. An unexpected behavior of Salmonella typhi. Dis Colon Rectum 1986;29:511-4.

12. Bharadwaj S, Anim JT, Ebrahim F, et al. Granulomatous inflammatory response in a case of typhoid fever. Med Princ Pract 2009; 18:239-41.

13. Cordova J, Shiloh R, Gilman RH, et al. Evaluation of molecular tools for detection and drug susceptibility testing of Mycobacterium tuberculosis in stool specimens from patients with pulmonary tuberculosis. J Clin Microbiol 2010;48:1820-6.

Affiliations: From the Division of General Internal Medicine, Department of Medicine (Cheung, Merkeley, Voyer), University of British Columbia, Vancouver, BC; the Department of Pathology and Molecular Medicine (Srigley), McMaster University, Hamilton, Ont.; the Division of Gastroenterology, Department of Medicine (Salh), University of British Columbia; and the Department of Pathology (Webber), University of British Columbia, Vancouver BC

Contributors: All of the authors contributed to the conception and design of the article, collected and interpreted the data, drafted the article and revised it for important intellectual content and approved the final version submitted for publication.

For more information on the diagnosis and management of typhoid fever, see Beaulieu AA, Boggild AK. CMAJ 2011;183:1740-5 (available at www.cmaj.ca/lookup/doi/10.1503/cmaj.101320). 